Meta

Journal des traducteurs

Translators' Journal

\title{
La linguistique comme science auxiliaire dans les disciplines juridiques
}

\section{Georges Mounin}

Volume 24, numéro 1, mars 1979

La traduction juridique

URI : https://id.erudit.org/iderudit/003676ar

DOI : https://doi.org/10.7202/003676ar

Aller au sommaire du numéro

Éditeur(s)

Les Presses de l'Université de Montréal

ISSN

0026-0452 (imprimé)

1492-1421 (numérique)

Découvrir la revue

Citer cet article

Mounin, G. (1979). La linguistique comme science auxiliaire dans les

disciplines juridiques. Meta, 24(1), 9-17. https://doi.org/10.7202/003676ar d'utilisation que vous pouvez consulter en ligne.

https://apropos.erudit.org/fr/usagers/politique-dutilisation/ 


\section{La linguistique comme science auxiliaire dans les disciplines juridiques}

Le thème est embarrassant. Non que la démarche du juriste s'interrogeant pour savoir s'il y a quelque chose à glaner dans les réussites d'une science sociale voisine de la sienne ne soit parfaitement légitime. Mais l'expérience a enseigné au linguiste, à travers les emprunts que l'ethnolographie, ou la critique littéraire (ou la sociologie, ou les arts plastiques, ou le cinéma) ont fait à la linguistique actuelle, que ces emprunts présentent des risques sérieux : soit d'être superficiels, soit d'être inadéquats, soit de se réduire parfois même à des réactions purement terminologiques de concepts archiconnus. La question ne se pose pas de proscrire ces emprunts, mais de faire qu'ils soient les bons, par un travail interdisciplinaire extrêmement vigilant.

Le noyau théorique essentiel de la linguistique structurale actuelle reste la phonologie. C'est la partie de la linguistique qui a résolu un problème théorique central sur lequel butait la description phonétique des sons du langage, et sur lequel elle butait d'autant plus qu'elle devenait plus scientifique, plus instrumentale, plus expérimentale. Par exemple, à tous les Français de 1890-1920, qui croyaient qu'il n'existait qu'un seul son [1] dans leur langue, les phonéticiens prouvaient de façon irrécusable qu'il en existait physiquement plusieurs, qui étaient articulatoirement et acoustiquement tout à fait distincts, et différents de façon sensible : le [1] de liberté qui était sonore, c'est-à-dire accompagné d'une vibration des cordes vocales, le [1] de simple, dit sourd, qui ne comportait pas ces vibrations, le [1] dit nasal, de chambranle, qui comportait, outre une vibration des cordes vocales, un écoulement partiel de l'air expiré par le nez. Fallait-il enseigner que la langue française comptait au moins trois [1] ? Comment expliquer alors que les Français n'en avaient pas conscience et, par exemple, employaient la même lettre pour représenter des sons scientifiquement distincts du point de vue physique ? Ou alors comment expliquer pourquoi ces [1] n'en faisaient linguistiquement qu'un seul ?

La phonologie, dans les années 1926-1938, résolut ce problème. Elle démontra que dans un son minimal, tous les traits qui le composent scientifiquement sur le plan de la description phonétique ne servent pas forcément dans la communication, ne sont pas distinctifs, ne sont pas pertinents. Par exemple la sour- 
dité du [1] de simple, la nasalité de celui de chambranle, pas plus que la sonorité du [1] de liberté ne sont, en français $\mathrm{du} \mathrm{xx}^{\mathrm{e}}$ siècle, des traits pertinents du phonème $/ 1 /$ : ils ne peuvent pas servir à opposer fonctionnellement dans la communication deux mots qui ne différeraient que par ces espèces de [1]. Le [1] de liberté ne peut pas être remplacé par celui de simple pour construire un autre mot de la langue française, tandis que si je remplace le son dental sonore [d] dans code par le son dental sourd correspondant [t], j'obtiens cote qui est un mot distinct. Il y a là deux phonèmes $/ \mathrm{d} /$ et $/ \mathrm{t} /$, dont l'opposition correcte assure la communication distincte des paires de mots comme code $\sim$ cote, rade $\sim$ rate, vide $\sim$ vile, etc.

C'est de ce type d'analyse, qui paraît relativement simple, voire évident aujourd'hui, que découlent tous les grands concepts opératoires qui caractérisent la linguistique contemporaine. L'importance centrale du concept de communication, par exemple, se substitue au concept de «langage comme expression de la pensée », non par hostilité à la pensée, ou à la philosophie, mais parce que ce sont - la phonologie le démontre - les lois de la communication (intercompréhension, économie) qui expliquent le fonctionnement et l'évolution des faits de langue. On aperçoit également l'importance liée des concepts de structure, de pertinence et de fonction : la sourdité et la sonorité de /t/ et de /d/ en français sont des traits structuraux pertinents de ces phonèmes français parce qu'ils ont une fonction de communication, celle d'assurer la distinction et l'intercompréhension de paires comme rateau $\sim$ radeau, tard $\sim$ dard, soute $\sim$ soude, etc. : tandis que les mêmes traits n'ont pas de fonction dans la communication en français pour le [1] de simple et le [1] de liberté. C'est le réseau des oppositions distinctives, c'est-à-dire pertinentes, des unités (phonologiques, lexicales, morphologiques, syntaxiques, stylistiques) d'une langue qui constitue les systèmes correspondants dont l'ensemble fait cette langue.

Y a-t-il quelque chose à prendre dans tout cela pour les sciences du droit ? La notion de communication, d'abord? Nous y reviendrons. Les notions d'oppositions distinctives (pertinentes), de structure, de fonction, de système? Sans doute la linguistique fournit-elle des illustrations claires, scientifiques, du contenu et du maniement de ces concepts qui sont chez elle opératoires. Mais si elle les a sinon découverts mais du moins popularisés, elle n'en a pas le monopole : ces mêmes concepts ont toujours été empiriquement à la base de toute activité scientifique de définition des objets, de classification en catégories, d'analyse et de description des ensembles d'objets liés entre eux par des relations, c'est-à-dire formant système. A cet égard donc, l'activité des linguistes peut servir d'exemple et non pas de modèle (au sens épistémologique actuel du terme), car il faut, dans chaque science, déterminer par des critères propres quelles sont les unités et les relations - les structures - qui sont pertinentes, c'est-à-dire qui ont une fonction (descriptive ou explicative) dans cette science.

II

Mais on pourra penser que, au-delà de ces généralités épistémologiques, la linguistique peut apporter aux sciences du droit, sur certains points précis, les 
outils tout prêts à fonctionner, en morphologie par exemple, en syntaxe. Des études sur la fréquence de l'indicatif, du futur, de l'impératif dans le texte du Code civil, ou des études sur le performatif dans les contrats, les témoignages, les formulaires, n'offrent-elles pas un grand intérêt? Observons d'abord que ces emprunts à la linguistique (concept de futur, d'indicatif, d'impératif) sont minces et marginaux, qu'ils pourraient être faits à partir de la vieille grammaire. En fait, il s'agit là de recherches, peut-être intéressantes, de stylistique, et de psycholinguistique dans la mesure où celle-ci s'intéresse aux effets produits par le message sur le récepteur. Stendhal n'a pas attendu Saussure, dont on n'a que faire ici, pour attirer l'attention sur des énoncés comme : « Tout condamné à mort aura la tête tranchée. \$ Le cas du performatif, en dépit de l'apparence, n'est pas différent. On sait que la philosophie anglo-saxonne récente appelle ainsi des énoncés (et surtout les verbes qui en sont les noyaux) tels que le fait même de les proférer constitue l'accomplissement de l'action qu'ils énoncent : Je jure que... Je promets de... J'ordonne... Je te conseille... Je m'excuse... Je vous souhaite... Je décrète... Je proclame... sont des performatifs (mais non : il jure, il promet, etc.). Il y a là une observation très fine sur l'un des mille et un usages du langage, un jeu de langage, comme disait Wittgenstein, très spécifique. Il se trouve que cet usage, ou ce jeu, sont particulièrement fréquents et intéressants dans la langue du droit. En décrire le mécanisme linguistique et social exact méritait d'être fait, mais il ne faut pas perdre de vue qu'il s'agit là encore d'un tout petit secteur, de la sémantique cette fois, dont l'exploitation épistémologique est délicate au demeurant (voir Austin, dans la Philosophie analytique, Édit. de Minuit, 1962, p. 271-281 ; aussi Benveniste, Problèmes de linguistique générale, Gallimard, 1966, p. 267-276).

\section{III}

Mais la lexicologie et la sémantique actuelles, elles, ne sauraient-elles apporter leur aide aux sciences du droit? Notons d'abord qu'il s'agit là du domaine où la linguistique postsaussurienne, structurale et fonctionnelle, et la linguistique chomskyenne, transformationnelle et générative, ont produit le moins de résultats assurés (cf. G. Mounin, Clefs pour la sémantique, Seghers, 1972). Ceci dit, l'étude du lexique de la langue du droit, et de tel droit spécialisé, n'en reste pas moins intéressante pour les juristes évidemment, dans la mesure où cette langue du droit constitue - par son lexique essentiellement - ce que Meillet définissait déjà en 1906 la «langue d'un groupe particulier 》 dans la société, un « vocabulaire spécial \$, une « langue spéciale \$; ou, comme on dit aujourd'hui, un « dialecte social ». Son article sur ce sujet (Linguistique historique et linguistique générale, Paris, Champion, 1926, p. 230-271, et surtout p. 241-252) reste d'ailleurs la meilleure introduction peut-être à ce problème.

Puisque l'ensemble des professeurs du droit constitue assurément un groupe particulier, par son activité même, qui a développé un lexique spécifique, nul doute qu'il n'y ait à inventorier ce lexique. Ici non plus la linguistique la plus en pointe n'apportera guère, sinon des perfectionnements lexicographiques. La vieille philologie enseignait déjà parfaitement à constituer des index de vocabulaire d'un texte 
et d'un auteur, et des concordances qui sont des index où les mots restent encadrés par leur contexte plus ou moins étendu. Sur ce point ce sont les ordinateurs qui représentent le grand progrès, par leur pouvoir de traiter rapidement d'immenses corpus de millions de mots de texte, et de livrer tout imprimés, à grande vitesse, des produits raffinés : index alphabétiques, index lemmatisés, concordances à contextes d'étendue variable en amont et en aval du mot, index de fréquence et de répartition des vocables, etc. Si un autre instrument doit être ajouté à cette panoplie c'est l'analyse du contenu, cadeau des progrès de la sociologie, qui permet une exploration révélatrice (même si elle reste grossière aux yeux du linguiste) des contenus sémantiques essentiels d'un texte (voir B. Berelson, Content Analysis, dans le Handbook of Social Psychology de C. Lindzey, Cambridge, 1954 - ouvrage toujours fondamental malgré les récents tâtonnements de l'analyse $d u$ discours, qui se présente généralement comme une hypothèse ou un projet encore confus dans ses procédures et ses résultats, pour dépasser l'analyse du contenu). Tout cela pouvant aboutir à des lexiques, à des dictionnaires de tel ou tel droit, ou bien à des études statistiques et stylistiques pour lesquelles $l e$ Vocabulaire du général de Gaulle de Cotteret et Moreau reste exemplaire (A. Colin, 1969).

\section{IV}

La sémantique proprement dite, de son côté, si peu développée soit-elle encore aujourd'hui et si conjecturales que demeurent ses hypothèses, peut offrir au droit l'exemple de quelques expériences suggestives. Le concept de champ sémantique, par exemple, pourra peut-être se révéler un instrument utile pour certaines investigations, mais ce n'est pas sûr et c'est aux juristes à le dire. En ce cas, l'analyse conduite par Jean Dubois sur le Vocabulaire politique et social en France (1869-1872) reste une bonne base de réflexion ${ }^{1}$. L'analyse thématique, qui est en réalité une espèce d'analyse de contenu conduite essentiellement à partir d'un index judicieusement utilisé, peut révéler dans un corpus donné les caractères inapparents. Par exemple, montrer que dans le Grand Meaulnes, presque toujours défini comme un roman «nocturne », le thème du jour et de la lumière est aussi fréquent que le thème de la nuit, fait qu'il resterait à expliquer mais qu'oblige à réfléchir sur un stéréotype critique accepté (voir Denise Millo, Mémoire de diplôme, Aix-en-Provence) - ou bien que la notion de poésie chez René Char et André Breton, bien qu'il s'agisse de deux poètes qui ont longtemps vécu ensemble et adhéré aux mêmes thèses, se révèle à l'analyse thématique presque totalement différente chez l'un de ce qu'elle est chez l'autre (Susan Wise, la Notion de poésie chez René Char et. André Breton, Aix, La Pensée Universitaire, 1967). Toujours en sémantique, le problème terminal est celui de la description des signifiés, c'est-à-dire un problème très voisin de celui de la description des concepts, en bref le problème (classique depuis Aristote et qui n'a guère progressế depuis, mếme chèz Leibniz) de la définition. Ce problème n'est pas propre à la linguistique ; il n'est pas différent, sur le plan théorique, pour la physique, la psychologie ou la philosophie. Tout au plus, la linguistique a-t-elle vérifié

1. . Voir ausși G. Mounin, Clefs pour la sémantique, avec une bibliographie. 
que le signifié d'un mot dans son ou ses usages n'est pas toujours constitué des mêmes traits pertinents ou sèmes que les traits définitoires ou réquisits qui définissent le concept associé à ce mot : la définition du signifié du mot « cheval * en français ne recouvre pas la définition du concept de cheval en zoologie.

Avant de quitter le domaine de la lexicologie et de la sémantique il faut sans doute évoquer une question, qui se pose pour le droit comme pour tout autre domaine social. A-t-on raison, scientifiquement, de parler de la langue du droit - ou de parler à son endroit de langue particulière, de langue spéciale ? Les formules de Meillet, qui éclairaient un domaine mal aperçu avant lui, risquent d'être ambiguës et de lancer les non linguistes sur une piste philosophiquement illusoire. Au sens propre il n'existe pas de langue du droit en soi mais seulement, à l'intérieur de la langue française, un vocabulaire du droit, et sans doute quelques tours syntaxiques spécifiques. Le moins curieux n'est pas que cette ambiguité ait été clarifiée par un non-linguiste, qui n'est autre que Staline, dans le débat fameux de 1950 contre Marr (voir les Cahiers du communisme, $\mathrm{n}^{0}$ 8, août 1950 , p. 13-36, et $\mathrm{n}^{\circ} 9$, sept. 1950 , p. 17-23). Il y démontrait que \& le rôle d'outil que joue la langue comme moyen de communication entre les hommes ne consiste pas à servir une classe au détriment des autres, mais à servir indifféremment toute la société, toutes les classes de la société »; et que, même si un groupe social ou une classe ont un lexique spécifique, voire des traits phonologiques ou syntaxiques caractéristiques, en ce qui concerne le fonds essentiel de la langue, on trouvera la même phonologie, le même lexique général, la même morphologie et la même syntaxe. Sinon il n'y aurait pas intercompréhension. On peut dire la même chose en d'autres termes : la a langue du droit », pour exprimer ce qu'elle a à dire, a besoin, non seulement du lexique spécifique du droit et de quelques particularités de syntaxiques propres, mais de toute la langue française. Il redevient de bon ton aujourd'hui de contester la position stalinienne, de soutenir qu'il y a dans un pays des langues de classe, ou que la langue a un caractère de classe : le texte stalinien n'ignorait pas les faits qu'on peut alléguer pour parler de «langue noble » et de «langue bourgeoise », de «langue prolétarienne » et de «langue campagnarde». Les faits sociolinguistiques que ces termes recouvrent (comme aussi celui de «langue juridique ») méritent d'être observés de près, mais ils n'enlèvent rien à l'existence prioritaire et fondamentale de la langue commune. Il y aurait certainement danger à vouloir faire d'une prétendue langue du droit une essence sui generis extérieure linguistiquement à la langue de tout le monde. Quand il n'y a pas intercompréhension entre les professions juridiques et les Français moyens il s'agit d'un phénomène social complexe, qui ne s'explique pas uniquement et peut-être pas principalement par l'existence du jargon juridique.

$\mathbf{V}$

Qu'est-ce que la stylistique, à son tour, peut offrir au juriste ? Si l'on admet qu'elle essaie d'être la science par laquelle on découvre ce que la façon de se servir d'une langue révèle sur l'émetteur lui-même, ou aussi la science qui étudie certains effets produits par certains messages sur les récepteurs, nul doute qu'il 
n'y ait place pour une stylistique du droit. Notons avant toute chose que la stylistique structurale et fonctionnelle est aussi peu avancée que la sémantique. Ceci posé, toutes sortes de méthodes (on pourrait en recenser une vingtaine, de Marouzeau jusqu'à Jakobson, et de Spitzer à Charles Mauron) se proposent soit pour fonder la stylistique génétique : celle qui révèle les motivations de l'émetteur - soit pour fonder la stylistique des effets : celle de l'action produite par le message sur le récepteur. C'est sans doute ici que devraient légitimement prendre place des études sur l'usage de l'impératif, du futur ou du présent de l'indicatif, selon les textes juridiques, par exemple.

\section{VI}

L'étude de l'évolution de la langue dans le temps, c'est-à-dire la linguistique diachronique, a naturellement sa place dans la panoplie d'instruments dont le droit peut légitimement disposer pour explorer sa propre histoire. Par exemple, est-ce que le mot propriété a le même sens à Rome (et pendant toute la durée du monde romain), dans une coutume rouennaise du xve siècle, à Paris aux xvIre$\mathrm{XVIII}^{\mathrm{e}}$ siècles, au $\mathrm{XIX}^{\mathrm{e}}$, au $\mathrm{XX}^{\mathrm{e}}$ ? C'est là un problème de sémantique historique qui n'a pas attendu la deuxième moitié du $x^{e}$ siècle pour être posé puisque c'est le domaine de la philologie la plus classique. La sémantique structurale actuelle n'apporte guère plus : là aussi, les ordinateurs peuvent seulement livrer à l'analyste des relevés totalement exhaustifs de tel mot dans le texte et, à la demande, un relevé à grande vitesse de tous ses contextes, délimités à volonté. L'analyse sémantique componentielle (en synchronie) ne livrera, sous le nom de sèmes du mot propriété (à telle ou telle époque), rien d'autre que la même chose à peu près que les traits définitoires du concept de propriété, ou réquisits, que la philologie aurait extraits, elle aussi, des concordances. L'intéressant ici sera toujours d'observer s'il y a désaccord entre le concept (définition légale du terme) et l'usage juridique du mot en contexte : cette discordance est révélatrice d'une distorsion entre la théorie et la pratique juridiques, entre le droit proclamé et le droit appliqué.

Un problème parallèle, quoique non historique, est posé par l'intercompréhension dans l'espace : l'ethnologie explore celui-ci, ou devrait l'explorer sur ce point, de la même façon que la philologie explore le temps. Par exemple ce problème $^{2}$ : est-ce que la notion recouverte par le mot propriété est la même à Tunis qu'à Paris en 1880, au moment de la conquête ? L'analyse montre que non : seules en 1880 sont considérées (par la législation arabe) comme posses"sions individuelles les parcelles cultivées (blé, jardins, vergers), non les pâturages collectifs de la tribu. L'application (consciente ou non ?) du concept de propriété français aboutit à considérer que le cheik, représentant (féodal) de la tribu, a le droit de vendre la terre collective. Pour parer à cette dépossession les fellahs sont conduits à mettre en culture les terres de pâturage mal drainées, ce qui entraîne les bas rendements et confirme les colons européens dans leur bonne conscience concernant la paresse, l'incapacité technique, l'inadaptabilité au pro-

2. Dont la substance et l'analyse m'ont été fournies par M. Claude Grenié, profesșeur à La Rochelle, à qui je suis heureux d'exprimer ici mes remerciements. 
grès des fellahs. Cet exemple, s'il est correctement interprété, montre bien que les mêmes mots (formellement bien traduits) ne recouvrent pas les mêmes choses (le même contenu sémantique). Comme la sémantique d'une langue c'est le contenu réel de toute sa civilisation, c'est tout le problème de la traduction des textes juridiques qui est illustré parfaitement, et soulevé ici.

\section{VII}

On peut croire, cependant, qu'à côté de ses apports techniquement limités, la linguistique fournirait au droit des principes généraux et des méthodes communes à toutes les sciences humaines - principes et méthodes qui sont aujourd'hui englobés et résumés par ce qu'on nomme le structuralisme. Rappelons obstinément que tout étant structuré (c'est-à-dire constitué d'éléments définis, réunis entre eux par des relations définies), le structuralisme est à sa place partout, en mathématique, en physique, en botanique, en chimie, etc. C'est d'ailleurs de ces domaines que viennent les notions de système, de taxinomie et d'organisme (métaphore biologique qui recouvrait et masquait le concept de structure, lequel s'en est dégagé lentement). Jean-Claude Gardin, pionnier de l'analyse documentaire (laquelle cherche toujours à produire ou à découvrir une structuration formelle et sémantique dans un domaine donné) a commencé par l'analyse structurale des poteries en archéologie ${ }^{3}$. La matière du droit doit donc pouvoir se prêter sans doute à l'analyse structurale : il y a sûrement une ou plusieurs structurations d'un code, comme l'a montré André Jean Arnaud ${ }^{4}$. Nul doute non plus que la ou les structurations qu'on découvre dans un code (ou dans tout autre corpus juridique) ne révèlent bien des caractéristiques (psychologiques, sociologiques, idéologiques, anthropologiques) inapparentes de ces documents. Tout le problème est ici de n'oublier jamais que l'analyse structurale d'un objet, quel qu'il soit, dépend totalement des critères de pertinence qu'on se donne pour extraire du corpus les unités qu'on va manipuler, ainsi que les relations qu'elles ont entre elles, et pour en démontrer l'existence objective. L'emprunt servile à la linguistique ne sera justifié que si les unités et les relations (juridiques) sur lesquelles on opère ont les mêmes propriétés que les phonèmes, les monèmes, les morphèmes, et le même type de relations entre eux. Si ce n'est pas le cas, si ce qu'on extrait du corpus n'est pas juridiquement pertinent, on n'aura emprunté à la linguistique que sa terminologie, passagèrement prestigieuse.

Nous avons déjà montré le péril qui s'attache à parler trop lâchement de la langue du droit (au lieu du vocabulaire de tel droit, ou du style spécifique de tel français juridique). Mais que penser de la formule assez fréquente aujourd'hui qui voudrait poser que « le droit est un langage » c'est-à-dire un système de communication identique aux langues naturelles humaines? Pour le linguiste, une langue est un système de communication doublement articulé, un code à deux étages : celui des unités distinctives ou phonèmes, et celui des unités si-

3. On the coding of geometrical shapes and other representations with reference to archaeological documents $\$$, Preprints of Papers for the International Conference of Scientific Information (Area 5), 1958, p. 75-87.

4. Essai d'analyse structurale dú Code civil, Paris, Librairie générale de Droit et de Jurisprudence, 1973. 
gnificatives ou monèmes, avec leurs règles propres de combinaison ${ }^{5}$. Tous les textes juridiques sont certainement des messages, ils assurent la communication - de type linguistique - d'un certain contenu - de type juridique. Mais quand, sous cette formule : " le droit est un langage », on veut sous-entendre (à la façon dont Lacan dit : l'inconscient est un langage, ou bien : est structuré comme un langage) que «l'acte juridique est un message» est-ce que l'extrapolation est défendable ? Je ne le crois pas : si l'acte juridique est un «message » parce qu'il a un "émetteur» et en général au moins un « récepteur», tout acte est un «message » et même tout produit est un «message ». Dewey disait déjà en ce sens que tout est langage, les gestes, les rites, les cérémonies, les monuments, les produits des beaux-arts et des arts industriels, parce qu'ils sont des signes par lesquels une civilisation donnée communique ce qu'elle est à d'autres générations, contemporaines ou postérieures.

Il y a ici une confusion fondamentale due à la polysémie du mot signe depuis au moins les Grecs. Lorsque Épicure dit que «le sang est un signe de la blessure, le mot signe n'a pas du tout le même sens que lorsque je dis : «Le mot sang est un signe linguistique constitué d'un signifiant phonique [sã], et d'un signifé « liquide visqueux, de couleur rouge, qui circule dans les vaisseaux à travers tout l'organisme, où il joue des rôles essentiels et multiples. ə Certes, dans les deux cas il y a une liaison entre un élément apparent, manifeste (ou plus manifeste que le second), et un élément non observable (ou moins directement observable, dont la présence est plus ou moins déduite du premier). Mais les règles par lesquelles je déduis que le sang est (peut-être) le signe de la blessure ne sont pas du tout semblables à celles qui unissent le signifiant et le signifié du signe sang. Dans ce dernier cas les règles de correspondance sont acquises à travers un code, acquis socialement, le même pour tous. Dans le cas du sang et de la blessure il s'agit d'un indice, il peut être perçu ou non en tant que tel, interprété exactement ou non. La signification linguistique est manifestée par un code ; la signification non linguistique d'un indice n'est pas manifestée par un code, mais interprétée par une science. Il ne suffit pas au Français moyen de se promener dans le Parthénon pour décoder la signification culturelle du temple grec : il lui faut étudier l'histoire et l'archéologie. De même ce sont toutes les sciences juridiques qui concourent, en en extrayant des indices significatifs pertinents, à faire dire par un corpus juridique, non pas sa signification linguistique évidente mais sa signification économico-sociologique, psychologique, idéologique, latente. Sans doute toute science vise-t-elle (à la limite asymptotique) à être une «langue bien faite», c'est-à-dire à construire de son domaine un modèle fait d'un stock total de tous les indices qu'on peut en extraire et des signifitions de tous ordres qu'on peut déduire de ceux-ci, de telle sorte qu'un appareil quelconque construit sur ce modèle donnerait à lire sur un cadran la signification

5. Ce serait une erreur de croire que cette vue - sans doute explicitée rigoureusement pour la première fois dans toutes ses conséquences épistémologiques par André Martinet - soit une vue propre à son auteur, isolée, discutable et discutée. En fait, sous une autre terminologie (duality of structure, duality of patterning, duality of levels), la linguistique anglo-saxonne a adopté ce point de vue. Tous ceux qui ont réfléchi au caractère spécifique des langues humaines naturelles, Vogelin, Hockett, Lyons, ont privilégié ce trait caractéristique fondamental. 
de tout fragment du corpus; et, en ce sens, la formule de Condorcet est vraie. Ainsi la température est-elle lisible (décodable) sur un thermomètre, et le poids d'un objet sur une balance, etc. Mais la complexité des «objets», et de leurs significations, dans les sciences humaines, nous fait toucher du doigt comme nous sommes encore loin de pouvoir fabriquer des modèles sûrs (de phénomènes relativement simples comme les attitudes sociales) permettant vraiment de lire la signification de ces phénomènes. Nous en sommes presque toujours au stade où nous cherchons à prouver que les indices que nous extrayons sont pertinents et sont exhaustifs. Nul doute que le droit ne soit une source d'indices considérable sur la collectivité qui l'a élaboré et qui le vit. Nul doute qu'il n'ait une signification économique, sociologique, culturelle, signification non lisible à première vue avec certitude, non manifeste. Nul doute non plus que cette signification (non linguistique) ne doive être déchiffrée. Mais, à mon avis, ce ne sera pas par la linguistique. L'apport réel ici de notre discipline aura été, non de proposer des modèles tout faits d'application mécanique au droit, mais d'avoir fourni un exemple stimulant pour réfléchir aux concepts épistémologiquement capitaux d'indice et de signe, de communication, de signification linguistique et non linguistique, de structure et de système - de pertinence aussi et peut-être surtout : car on ne répétera jamais assez que si l'on extrait d'un corpus des indices non pertinents ou statistiquement non représentatifs, et si on les combine par des interprétations discutables, on n'aura construit qu'une pseudo-structure du corpus, plus représentative de l'idéologie du chercheur que de celle de l'objet juridique étudié. 